Esta sección contiene los artículos originales de las Revistas de Pediatría de las Sociedades de Pediatría del Cono Sur seleccionados para ser publicados por los países integrantes durante el año 2012

\title{
Daño celular en una población infantil potencialmente expuesta a pesticidas
}

\author{
BENÍTEZ-LEITE S. ${ }^{1}$, MACCHI ML. ${ }^{1}$, FERNÁNDEZ V. ${ }^{2}$, FRANCO D. ${ }^{2}$, \\ FERRO EA. ${ }^{3}$, MOJOLI A. ${ }^{2}$, CUEVAS F. ${ }^{2}$, ALFONSO J. ${ }^{2}$, SALES L. ${ }^{2}$ \\ 1. Cátedra de Pediatría, Facultad de Ciencias Médicas (FCM), Universidad Nacional de Asunción (UNA). San Lorenzo, Paraguay. \\ 2. Laboratorio de Mutagénesis Ambiental, Facultad Ciencias Exactas y Naturales (FaCEN) UNA. \\ 3. Cátedra de Bioquímica FCM-UNA.
}

\begin{abstract}
Cell damage in a pediatric population potentially exposed to pesticidas

Introduction: Pesticides can cause acute or chronic effects on human health. Many pesticides can cause damage to genetic material. These changes in genetic information have been associated with an increased risk of cancer. Objective: To investigate damage to genetic material in a population of children potentially exposed to pesticides in the environment. Method: The study design was observational and cross sectional. We studied 48 children potentially exposed to pesticides and 46 unexposed children. Samples of the oral mucosa were obtained to determine damage to genetic material using micronucleus frequency (MN). Results: Higher average micronucleus counts were found in the group potentially exposed to pesticides ( $5.1 \pm 2.9 v s 1.8 \pm 2.0$, $\mathrm{p}<0.0001)$, along with a higher average of binucleate cells ( $3.5 \pm 2.7$ vs $1.4 \pm 1.4 \mathrm{p}<0.0001)$, higher frequency of karyorrhexis (18.2 \pm 18.4 vs $5.8 \pm 18.4, \mathrm{p}<0.004)$ and pyknosis $(24.8 \pm 18.0$ vs $17.1 \pm 8.3 ; \mathrm{p}<0.03)$. Of the children potentially exposed to pesticides, $40 \%(19 / 47)$ had an exposure time of 6 years. Conclusion: This study provides evidence of genetic damage in the population potentially exposed to pesticides in the environment.
\end{abstract}

(Key words: Mutagens, genotoxins, pesticides, children, child, environment).

Pediatr (Asunción) 2010; 37 (2): 97-106.

\section{RESUMEN}

Introducción: Los pesticidas pueden producir efectos agudos o crónicos en la salud humana. Muchos de ellos pueden provocar daño en el material genético. Esta modificación en la información genética se ha relacionado con un alto riesgo de padecer cáncer. Objetivo: Indagar el daño en el material genético de una población infantil expuesta potencialmente a pesticidas en el ambiente. Método: El diseño metodológico fue de tipo observacional y transversal. Participaron en el estudio 48 niños expuestos potencialmente a pesticidas y 46 niños no expuestos. Se obtuvo muestra de la mucosa bucal para determinar daño en el material genético a

Esta investigación se realizó en el marco de la convocatoria 2009 para proyectos de investigación de la Dirección General de Investigaciones Científicas y Tecnológicas de la Universidad Nacional de Asunción.

Correspondencia a:

Dra. Stela Benítez-Leite

E-mail: stelabenitez@yahoo.com.ar 
través de la frecuencia de micronúcleos (MN). Resultados: Se encontró en el grupo expuesto potencialmente a pesticidas un promedio mayor de micronúcleos (5,1 $\pm 2,9$ vs 1,8 $\pm 2,0$; p < 0,0001), un promedio mayor de células binucleadas (3,5 $\pm 2,7$ vs $1,4 \pm 1,4$; p < 0,0001), mayor frecuencia de cariorrexis $(18,2 \pm 18,4$ vs 5 ,8 $\pm 18,4$; $\mathrm{p}<0,004)$ y picnosis $(24,8 \pm 18,0$ vs 17,1 \pm 8,3; p < 0,03). El 40\% (19/47) de los niños expuestos potencialmente a pesticidas tuvieron un tiempo de exposición de 6 años. Conclusión: Esta investigación aporta evidencias de daño genético en la población expuesta potencialmente a pesticidas en el ambiente.

(Palabras clave: Genotoxicidad, pesticidas, niños, medio ambiente).

Pediatr (Asunción) 2010; 37 (2): 97-106.

\section{ESTE ARTÍCULO SE PUEDE ENCONTRAR EN EXTENSO EN WWW.SCIELO.IICS.UNA.PY EN PEDIATR (ASUNCIÓN), 37 (2): 97-106, 2010}

